\title{
El video como herramienta de elaboración de trabajos del alumnado
}

\author{
Quintela del Río, Alejandro \\ Departamento de Matemáticas, \\ Facultad de Fisioterapia - Facultad de Informática, UDC
}

\section{RESUMO}

En este trabajo se expone la experiencia del video digital como herramienta de aprendizaje virtual para el alumnado, dentro de los nuevos sistemas de educación en el entorno universitario. En vez de elaborar trabajos en el clásico formato de texto, para ser presentado en PowerPoint, Prezi, Word o similar, los alumnos han desarrollado y editado videos digitales, en los que ellos mismos presentan el trabajo a realizar y explican los pasos seguidos a través de grabaciones del ordenador.

PALABRAS CLAVE: Video digital, tecnologías de la educación, nuevos medios, aprendizaje virtual.

\section{CITA RECOMENDADA:}

Quintela del Río, A. (2018). El video como herramienta de elaboración de trabajos del alumnado. En E. de la Torre Fernández (ed.) (2018). Contextos universitarios transformadores: retos e ideas innovadoras. II Xornadas de Innovación Docente. Cufie. Universidade da Coruña (pág. 101-110).

DOI capítulo: https://doi.org/10.17979/spudc.9788497496780.101

DOl libro: https://doi.org/10.17979/spudc. 9788497496780 


\section{ABSTRACT}

This work presents the experience of digital video, as a virtual learning tool, for students within the new education systems in the university. Instead of elaborating works in the classic format (text), for presentation by means of computer programs as PowerPoint, Prezi, Word or similar, the students have developed and edited digital videos, in which they themselves present the work to be done, and they explain the followed steps through computer recordings.

KEY WORDS: Digital video, Educational Technology, New media, e-Learning. 


\section{INTRODUCCIÓN}

La docencia universitaria se ha desarrollado fundamentalmente a través de clases expositivas y magistrales, trabajando en pequeños seminarios 0 a través de una bibliografía básica del curso. Generalmente, al final de curso, los estudiantes son evaluados sobre la base de una prueba escrita, o bien a través de trabajos desarrollados en grupo y presentados en un formato de texto escrito. Santos Guerra (2015) indica que los profesores y los alumnos son meras herramientas del proceso de evaluación. A lo largo del mismo, el alumno permanece en segundo plano y el protagonista indiscutible, el profesor, se encarga de organizar y planificar el aprendizaje.

Sin embargo, esta situación está cambiando en las aulas universitarias, ya que se han desarrollado metodologías pedagógicas centradas en el aprendizaje de los estudiantes, y que permiten una mayor participación en el proceso de enseñanza, mediante las denominadas "metodologías activas". Uno de ellas es el Aprendizaje Basado en Proyectos (ABP). A partir de esta metodología, el alumno se convierte en protagonista del proceso de enseñanza y aprendizaje, y el profesor se convierte en mediador o guía de este proceso (Vizcarro et al., 2008). Algunos de los puntos clave del ABP son que proporciona a los estudiantes contextos reales de aprendizaje; les involucra en el diseño, la resolución de problemas y, sobre todo, en la toma de decisiones y la actividad investigadora que conlleva (Grahame, 2011).

Esta metodología ha sido ampliamente aceptada en diferentes entornos universitarios, tanto de ciencias como de letras. Asimismo, estos enfoques pedagógicos han sido implementados en diferentes contextos internacionales $\mathrm{El} A B P$ es un enfoque educativo que enseña a los estudiantes a trabajar en colaboración como grupo para encontrar soluciones a un problema real. También se ha encontrado que ayuda a los estudiantes a adquirir un aprendizaje, ya que les permite trabajar de manera relativamente autónoma y lograr mejores resultados en relación con el problema planteado (Thomas et al., 1999). Podemos destacar que el ABP debe ser una de las herramientas fundamentales para el desarrollo de competencias (creatividad, resolución de problemas, capacidad de investigación, trabajo colaborativo, motivación y uso de redes 
sociales, entre otras) que se promoverán en los sistemas educativos del siglo XXI. Comparando los entornos de enseñanza tradicionales con otros en los que se aplica el ABP, se ha demostrado que los estudiantes muestran mejores habilidades para resolver problemas y aparecen como más comprometidos, más autosuficientes y más atentos (Walker et al., 2009). Hoy en día, el video se ha convertido en una herramienta de comunicación fundamental, y ha adquirido una enorme importancia en la tarea de transmisión de contenidos docentes. No hay más que ver cómo han evolucionado los canales de este ámbito en Youtube, en todo tipo de páginas académicas y en las propias webs de las universidades. Se ha comprobado que los jóvenes de hoy en día tienen un concepto visual mucho más desarrollado, debido al tiempo que han estado expuestos a las nuevas tecnologías de la comunicación desde la primera infancia (Moreno, 2006). Asimismo, se ha demostrado que, en el proceso de aprendizaje, la cognición de la visión espacial es de una gran importancia (Wurdinger \& Qureshi, 2015). Los estudiantes quieren crear utilizando las herramientas de su tiempo, trabajar con sus compañeros tomando sus propias decisiones y compartiendo el control, no sólo en clase, sino también conectando con otros jóvenes de todo el mundo para comparar y competir entre sí. Además, buscan que el conocimiento adquirido se aplique inmediatamente a algo real (Prensky, 2010). En el nuevo paradigma educativo, el aprendizaje a través del trabajo cooperativo es relevante, en el que los sistemas multimedia ofrecen valiosas oportunidades, tanto en el aula como en Internet.

Las Tecnologías de la Información y la Comunicación (TIC) reúnen elementos y técnicas utilizadas en el tratamiento y transmisión de la información; son herramientas que pueden ser utilizadas para la construcción de materiales didácticos, facilitando el aprendizaje y el desarrollo de habilidades, ya que requieren que los estudiantes movilicen su pensamiento crítico y analítico, interactuando con ellos (Miranda et al., 2012), desarrollando su capacidad de respuesta a los retos y problemas que plantea cada materia y su futuro empleo. Las TIC se están convirtiendo en herramientas comunes en las escuelas públicas de los países desarrollados, extendiéndose a los sistemas educativos de los países en desarrollo. Estas 
tecnologías permiten que los procesos cognitivos jueguen un papel más importante en el aprendizaje de materiales educativos y científicos complejos, en respuesta a la evolución natural de los métodos de aprendizaje y a la interrelación de los jóvenes como resultado del rápido desarrollo de las tecnologías de la información y las comunicaciones.

Por otra parte, no debemos olvidarnos que esta forma de utilización de las TIC propicia alcanzar los últimos niveles de la taxonomía de Bloom para la era digital (Chursches, 2007), que es la categoría "crear", es decir, aquella en la cual el estudiante se centra en la producción original de contenidos, y no olvidándonos de sus potencialidades para que los alumnos adquieran algunas de las competencias que desde el Espacio Europeo de Educación Superior se señalan que deben alcanzar (Herrero, 2014).

Ahora bien, esta utilización no se refiere a la realización de acciones no planificadas, sino más bien al diseño de acciones educativas altamente planificadas donde los alumnos deberán de realizar una serie de aspectos, como son: Interaccionar con la información, identificarla para adaptarla a su proyecto educativo y de investigación; organizarla en un guion específico adaptado al contexto del medio seleccionado, aprendizaje de la tecnología a utilizar, y la producción del medio seleccionado. El proyecto será mucho más enriquecedor desde el momento en que se realice a través de experiencias compartidas en grupos.

\section{DESCRICIÓN DA EXPERIENCIA}

La experiencia realizada se llevó a cabo en la asignatura "Documentación y Estadística Sanitaria", obligatoria de cuarto curso del grado en Fisioterapia de la Universidad de A Coruña (Campus Sanitario de Oza). Se dividió el alumnado en grupos de 304 estudiantes, y los trabajos consistían en el análisis estadístico de un conjunto de datos. El proceso de realización del mismo consistió de los siguientes pasos:

- Toma de datos (encuesta) relacionados con la asignatura.

- Análisis de datos mediante el paquete estadístico SPSS (IBM, 2017)

- Presentación de resultados e interpretación. 
La realización concreta del trabajo y su presentación debía ser a través de un video corto (entre 20-30 minutos de duración). Debería consistir en la realización secuencial de los siguientes apartados:

- Una presentación: tarea que puede realizarse directamente a través del teléfono móvil.

- Grabación del trabajo realizado en el ordenador: Puede utilizarse el software Camtasia (Camtasia, 2017) (de pago), o bien una herramienta opensource como Captura (Captura, 2017).

- Edición del trabajo (cortar, pegar trozos, insertar audio, insertar música...):_Puede utilizarse el software Camtasia, o bien una herramienta de software libre como Shotcut (Shotcut, 2017).

- Conversión del fichero realizado (si el video resultante es muy grande, puede transformarse a un fichero .mp4, por ejemplo, sin perder excesiva calidad):

Puede realizarse a través de un programa convertidor de formatos como Format Factory (Format Factory, 2017), que es gratuito.

- Alojamiento del archivo final en un espacio online (Dropbox, Google Drive, OneDrive...): El video generado puede resultar de un tamaño excesivo para ser enviado al profesor directamente por e-mail, por lo que recomendamos el alojamiento del mismo en un almacenamiento gratuito en la nube.

- Envío de enlace al profesor por mail: Del trabajo resultante se le enviará el enlace correspondiente al profesor por e-mail para que él mismo lo descargue para la corrección del trabajo.

\section{RESULTADOS}

Dividimos los resultados del proceso en dos apartados de ventajas observadas, primero para el alumnado y después para el profesorado.

Ventajas observadas para el alumnado. 
- Utilización de herramientas nuevas de edición de video digital, que se revelan muy útiles para otras asignaturas 0 en futuras aplicaciones, tal vez fuera de la universidad (posteriores trabajos, CV online en video...) (Borko et al., 2012).

- Mayor compromiso de trabajo, tanto grupal como individual, en un entorno colaborativo (Brouwer, 2007).

- Entorno de trabajo adaptado al ambiente al que alumno está habituado. La familiaridad con el mismo le produce un menor estrés de trabajo y la satisfacción con los resultados del mismo pueden ser más relevantes (Wurdinger et al., 2015).

- La tarea de edición de video digital supone un complemento añadido a la tarea de aprendizaje básico, por lo que el resultado es una mayor comprensión de los conocimientos propios de la asignatura y de las competencias a adquirir (Bayran, 2012).

Las ventajas para el profesorado también son varias, al realizarse la corrección de manera más rápida, pudiendo también comprobar con mayor profundidad el grado de aprendizaje del alumno. La corrección y puntuación se convierte, por tanto, en una tarea menos monótona y menos automatizada (Brouwer, 2007).

\section{CONCLUSIÓNS}

Este trabajo ha presentado nuestras experiencias estableciendo un marco de trabajo nuevo en un entorno activo para la enseñanza y el aprendizaje. Los puntos fuertes son que proporciona un enfoque sistemático para facilitar un mayor compromiso de los estudiantes con los temas de la asignatura, y permite un enfoque extraacadémico, por cuanto en la mayoría de las ocasiones el banco de datos con el que se realiza el trabajo de análisis estadístico se obtiene por medio de encuestas directas a personas fuera del ámbito universitario. 
Una posterior mejora y posible ampliación del estudio podría consistir en una coordinación horizontal entre los distintos grupos de alumnos, de cara a realizar una encuesta más amplia, de modo que cada uno de los grupos constituyese una parte de lo que podría ser un gran documento de trabajo de fin de curso. Creemos que trabajar de esta manera podría mejorar el beneficio del conocimiento producido en términos de los proyectos individuales.

Quizá uno de los principales problemas de una coproducción es que requiere mayor tiempo y esfuerzo para iniciar un proceso que podría resultar muy provechoso, tanto en términos de demanda de recursos como de desconocimiento. Un reto clave para la coproducción consiste en facilitar y racionalizar la identificación de proyectos aplicados y en asociar a los estudiantes con las partes interesadas, lo que conllevaría un grado de implicación del profesor todavía mucho mayor en la dirección general del proyecto.

En cualquier caso, nuestras experiencias sugieren que la producción de trabajos de clase mediante herramientas de video digital es eficaz, ya que permite una mayor implicación con materiales de trabajo actuales y de interés para los estudiantes. Creemos que los resultados son positivos tanto para los alumnos como para el profesorado, por todo lo expuesto en el punto anterior. Ello nos anima a seguir avanzando en el conocimiento y uso de este tipo de experiencias, en el que los trabajos de clase puedan ser presentados en formato digital, u otro formato innovador y alternativo al clásico de texto, que a la vez pueda ir parejo al desarrollo de nuevas tecnologías, y permita añadir interactividad a las clásicas herramientas de presentación del trabajo en formato estático.

\section{REFERENCIAS}

- Bayram, L. (2012). Use of online video cases in teacher training. Procedia-Social and Behavioral Sciences, 47, 1007-1011.

- Borko, H., Jacobs, J., Eiteljorg, E., \& Pittman, M. E. (2008). Video as a tool for fostering productive discussions in mathematics professional development. Teaching and Teacher Education, 24, 417-436. 
- Brouwer, N. (2007). Imaging teacher learning: A literature review on the use of digital video for preservice teacher education and teacher professionalization. Nijmegen: ILS Graduate School of Education.

- Camtasia (2017). Software de edición de video. Tech-Smith. https://www.techsmith.com/video-editor.html

- Churches, A. (2007). Educational Origami, Bloom's and ICT Tools. Recuperado de:http://edorigami. wikispaces.com/Bloom\%27s+and+ICT+tools (20/06/2013).

- Format Factory (2017). Convertidor multifuncional de medios de comunicación. https://www.pcfreetime.com

- Grahame, S. D. (2011). Science education in a rapidly changing world. Hauppauge, New York

- Herrero, R. (2014). El papel de las TIC en el aula universitaria para la formación en competencias del alumnado. Pixel-Bit. Revista de Medios y Educación, 45, 173-188.

- IBM Corp. Released 2017. IBM SPSS Statistics for Windows, Version 24.0. Armonk, NY: IBM Corp.

- Moreno, M. E. (2006). Las TIC y el Desarrollo del Aprendizaje en Educación Inicial. Revista Electrónica de Humanidades, Educación y Comunicación Social, $1^{a}$ edición, año 1, 1-11.

- Prensky, M. (2010). Teaching Digital Natives: Partnering for Real Learning. Corwin Press, California, USA.

- Sachin, M. (2017). Captura. https://mathewsachin.github.io/Captura/

- ShotCut. Plataforma opensource de edición de video. https://www.shotcut.org/

- Thomas, J.W., Mergendoller, J.R. \& A. Michaelson (1999). Project Based Learning: A handbook for middle and high school teachers, Novato, CA, The Buck institute for Education. 
- Vizcarro, C., \& Juárez, E. (2008). ¿Qué es y cómo funciona el aprendizaje basado en problemas?, in El aprendizaje basado en problemas en la enseñanza universitaria, bv Universidad de Murcia, Servicio de Publicaciones, pp. 17-36 Murcia, España .

- Walker, A., \& Learv, A. (2009). Problem based learning Meta Analysis: Differences across problem types, implementation types, disciplines and assessment levels, The Interdisciplinarv Journal of Problem-Based Learning, 3(1), 12-43

- Wurdinger, S. \& Qureshi, M. (2015), Enhancing College Students' Life Skills through Project Based Learning, Innovative Higher Education, 40(3), 279-286.

- Xu, Y., \& Liu, W. Q. (2010). A project-based learning approach a case study in China. Asia Pacific Education Review, (3), 636-370. 